\title{
Model Predictive Control for Improving Operational Efficiency of Overhead Cranes
}

\author{
Zhou Wu · Xiaohua Xia · Bing Zhu
}

\begin{abstract}
Model predictive control (MPC) has been successfully applied to many transportation systems. For the control of overhead cranes, existing MPC approaches mainly focus on improving the regulation performance, such as tracking error or steady-state error. In this paper, energy efficiency as well as safety is newly considered in our proposed MPC approach. Based on the system model designed, the MPC approach is applied to minimize an objective function that is formulated as the integration of energy consumption and swing angle. In our approach, promising results in terms of low energy consumption and small swing angle can be found, whilst the solutions obtained can satisfy all practical constraints. Our test results indicate that the MPC approach can ensure stability and robustness of improving energy efficiency and safety.
\end{abstract}

Keyword: overhead crane; model predictive control; open-loop control; anti swing; energy efficiency

\section{Introduction}

Overhead cranes are widely used to transport heavy or hazardous loads in factories and harbors. For a transportation task, the crane is expected to arrive the end quickly with acceptably small swing. However it is difficult to meet such requirements of time efficiency and safety in crane control, as the crane is an underactuated system with one control input (actuating force) but two degrees of freedom (motion of the trolley and swing of

Zhou Wu Xiaohua Xia · Bing Zhu

Department of Electrical, Electronic and Computer Engineer-

ing, University of Pretoria, Pretoria, SA

Tel.: +27124204341

E-mail: wuzhsky@gmail.com, xxia@up.ac.za,

bing.zhu@up.ac.za the payload). Positioning accuracy and anti-swing are two basic requirements, which are highly correlated in the crane operation. Researchers in the communities of mechatronics and control have made a lot of efforts on designing effective and efficient control methods for the underactuated crane $[3,15,18,32]$. The difficulty of control arises from the strong state coupling between motion of the trolley and swing of the payload. The swing not only affects operational efficiency, but also brings potential risks of damaging the payload or surrounding objects. Therefore, the first task in crane control is to analyze such kinematic coupling behavior. Researchers often separate the design of controller into an anti-swing part and a positioning part, and then combine the two parts for achieving required performance.

Many previous works have considered improving regulation performances of overhead cranes. Existing strategies can be generally grouped into three categories: optimal control, input shaping and feedback control. Optimal control was first proposed as an open-loop strategy to design the optimal sequence of control. Motion trajectories obtained are optimal in terms of some preferred objectives, such as the steady-state error or transportation time $[17,23]$, while satisfying practical and physical constraints. Input shaping is a command generation method that aims to limit residual swing [8]. The swing induced by the first part of the command is canceled by the swing induced by the following part of the command. Input shaping is implemented in real time by convolving the command signal with an impulse sequence [26]. Note that optimal control and input shaping are not suitable for some applications with large system uncertainties and external disturbances as they do not have closed-loop mechanisms.

For the third category, feedback control is the most commonly used strategy for underactuated systems. Based 
on real-time measured information, such as position, velocity and swing angle, the control input is adjusted to reduce the error between actual and referenced states. Proportional derivative (PD) control $[4,6]$, sliding-mode control [19,31,21], fuzzy control [13,2] and adaptive control [34] have all been applied to overhead cranes in the fold of feedback strategies. Generally, feedback strategies can be divided into two types, tracking and non-tracking strategies. Firstly, trajectories are designed to maximize operation efficiency under some physical constraints[28]. Then feedback control is employed to track the planned trajectories in the environment with uncertainties and disturbances $[5,22,16]$. In these tracking strategies, additional residual swing will be caused during the tracking process even though the planned trajectories are optimally designed to suppress residual swing. Secondly, non-tracking strategies have been proposed to regulate the control performance in real time $[34,27,12,30]$. These methods skip the step of trajectory planning but still achieve positioning accuracy and Lyapunov stabilization. Due to lack of planning mechanisms, it is difficult to optimize operational efficiency for this type of feedback strategies. To overcome these weakness aforementioned, a model predictive control approach is proposed as a feedback strategy.

In this paper, model predictive control (MPC) is developed for crane control, in which trajectory planning is not required but operational efficiency (in terms of energy and safety) can be optimized. MPC has emerged since the early 1970s, and MPC has been successfully applied particularly in the process control. MPC is a feedback control strategy that uses an explicit model of plant to predict the future response of plant over a finite horizon. The goal of MPC is to compute a future control sequence in the defined horizon by minimizing a cost function, which is subject to a set of constraints both in the control actions and the plant outputs [7,33]. Only "the first part" of the sequence is applied to control at the next state. Theoretical properties such as stability and robustness of MPC have been studied by many authors since the early work [11]. Up to the present, MPC has become one of the most popular multivariable control algorithms in various industries, including chemical engineering, food processing, aerospace application and recently in power systems [24,33,20]. This is due to its facility of handling constraints, its ability of using simple models, and its closed-loop stability and inherent robustness in many applications. In the transportation field, MPC has been employed to solve the path-tracking problems of terrestrial autonomous vehicles [25] and heavy-haul trains [35]. Researchers have also applied MPC to boom cranes [1] and gauntry cranes [10] for tracking and anti-swing. However, exist- ing MPC approaches for cranes only consider minimizing tracking error and steady-state error. They have neglected two important issues, i.e., energy efficiency and safety, which turn out to be significantly urgent when a large number of cranes have been equipped in some international industrial fields. To the best of our knowledge, little work has been done to minimize the swing risk while most work only considered the swing as a constraint of control. Besides the swing risk, the control sequence is also related to the profile of power, as well as the total energy consumption. However, as such relation is not clear yet, energy consumption has seldom been considered in crane control.

In this paper, two objectives, energy efficiency and safety, are evaluated in our proposed MPC approach. The contributions of this paper include the following four aspects. Firstly, in our MPC approach the two objectives have been integrated based on a discrete-time model of crane. Our approach can deliver promising performance in terms of minimal energy consumption and swing. Secondly, our MPC approach does not require a reference trajectory, which reduces the workload of controller. Thirdly, most practical and physical constraints, such as zero residual swing, maximal velocity and acceleration, are satisfied in our MPC approach. Fourthly, due to the closed-loop structure of MPC, the proposed approach has good robustness when the crane system experiences disturbances. The proposed MPC approach has been compared with the open-loop control approach in the simulation part. The comparative study is shown that our MPC approach can deliver better control performance than the open-loop control, in terms of energy consumption, swing and robustness.

The rest of this paper is organized as follows. Section II presents the dynamical model of overhead cranes. The discrete-time model of overhead cranes is deduced in Section III. The proposed MPC approach to optimize the energy efficiency and the safety is given in Section IV. The comparative results are shown in Section V. Section VI concludes this paper.

\section{Dynamic Model of Overhead Cranes}

The structure of an overhead crane can be illustrated as shown in Figure 1, where the trolley moves on the horizontal bridge and the payload is connected with a constant-length rope. Let $p(t), \theta(t)$ and $F(t)$ denote the trolley's position, the payload's swing angle and overall force on the trolley respectively. In this paper, bridge deformation, air resistance as well as stiffness and mass of the rope is neglected, and the load is considered as a point mass. Moreover, as this study only focuses on the control of horizontal transportation, hoisting and 


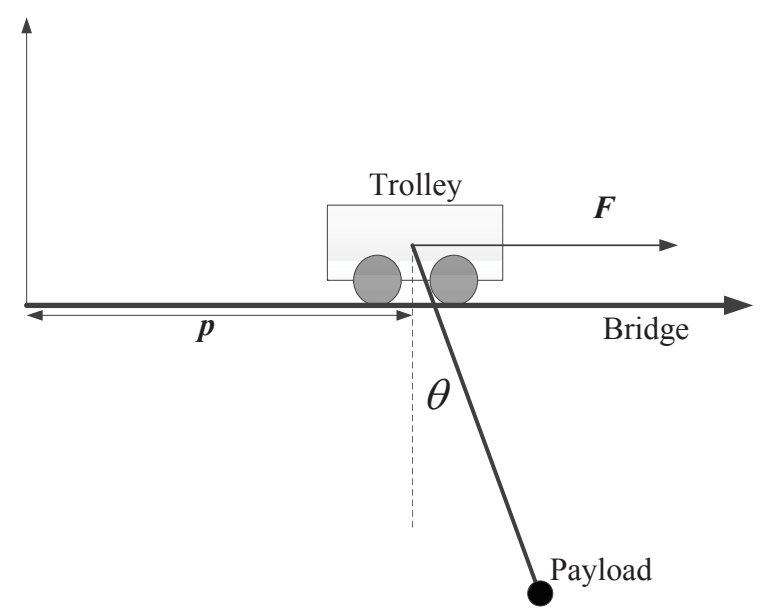

Fig. 1 Two-dimensional overhead crane system

lowering of the payload are not considered. Then the overhead crane system with constant rope length can be described as follows:

$(M+m) \ddot{p}+m l \cos \theta \ddot{\theta}-m l \sin \theta \dot{\theta}^{2}=F$,

$m l^{2} \ddot{\theta}+m l \cos \theta \ddot{p}+m g l \sin \theta=0$,

where $M$ and $m$ denote masses of the trolley and the payload, respectively. $l$ is the length of the rope; $g$ is the gravitational acceleration. The overall force $F$ is composed of the actuating force $F_{a}$, the friction $F_{r}$ and the disturbance $d$ as

$F=F_{a}-F_{r}+d$,

Motivated by the friction models in $[14,29,9]$, this paper employs a similar nonlinear friction model as

$F_{r}=f_{r 0} \tanh (\dot{p} / \xi)+k_{p} \dot{p}+k_{r}|\dot{p}| \dot{p}$,

where $f_{r 0}, k_{p}$ and $k_{r} \in \mathbb{R}$ are friction-related parameters and $\xi \in \mathbb{R}$ is a static friction coefficient, which can be obtained from off-line experimental analysis. In the righthand side, the first component is the Coulomb friction, the second one is the damping effect of the trolley, and the third part is an approximation of other effects. Note that the small friction caused by the payload's swing is neglected in the above model.

The crane dynamics consist of the actuated part (Eq. (1)) and the underactuated part (Eq. (2)). The latter part is the system kinematics that defines the coupling behavior between the trolley's acceleration $\ddot{x}(t)$ and the payload's swing angle $\theta(t)$. The main difficulty in controlling the overhead crane lies in the handling of such coupling behavior. When the swing angle is small enough $\left(\theta(t)<5^{\circ}\right)$, the kinematic equation (2) can be linearized with the approximations of $\cos \theta \simeq 1$ and $\sin \theta \simeq \theta$. The approximated linear kinematics can be obtained as

$l \ddot{\theta}+\ddot{x}+g \theta=0$.

In the evaluated time interval $[0, T]$, the crane is required to arrive at the destination without residual swing. Therefore, several principles must be satisfied according to the physical and practical situations in crane control.

Principle 1: The trolley reaches the desired location $p_{d}$ at the end of the period. The final states must ensure that the trolley is static with no swing and that it can be lowered immediately as

$p(T)=p_{d}, \dot{p}(t)=0, \theta(T)=0, \dot{\theta}(T)=0$.

Principle 2: During the horizontal transportation, the velocity and acceleration of the trolley must be limited in certain ranges as

$\left\{\begin{array}{l}0 \leq \dot{p}(t) \leq v_{m}, t \leq T \\ |\ddot{p}(t)| \leq a_{m}, \quad t \leq T\end{array}\right.$

where $v_{m}$ and $a_{m}$ are the permitted maximum of velocity and acceleration, respectively.

Principle 3: The payload swing during the transportation must be limited within a safe range as

$|\theta(t)| \leq \theta_{m}, t \leq T$,

where $\theta_{m}$ is the permitted maximum of swing amplitude.

In the approach of control system, the dynamic model of overhead crane can be rewritten as a multiple input multiple output (MIMO) state-space model. Denote the system state as $x \triangleq\left[p-p_{d}, \dot{p}, \theta, \dot{\theta}\right]$, the system input as the acceleration $u \triangleq \ddot{p}$, the system output as $y \triangleq x$. According to Eq. (5), the linear state-space equation can be expressed as

$\left\{\begin{array}{l}\dot{x}=\left[\begin{array}{cccc}0 & 1 & 0 & 0 \\ 0 & 0 & 0 & 0 \\ 0 & 0 & 0 & 1 \\ 0 & 0 & -w_{n}^{2} & 0\end{array}\right] \\ y=x\end{array}\right.$

where $w_{n}=\sqrt{g / l}$ is the natural frequency of system. Note that the continuous steady-space models have been used in many linear control methods [5, 12], in which the system has been proven controllable and stable. 


\section{Discrete-time Model of Overhead Cranes}

Many control approaches are implemented on discrete systems, where at each sampling instant MPC uses the current state of plant to compute the input for the next control period. Therefore, the continuous system is first discretized by a sampling period $t_{0}$, and $N=T / t_{0}$ is the total number of samples. The discrete-time model of cranes can be formulated as Eq. (10) and (11).

$$
\begin{aligned}
& (M+m) a(n)+m l \cos \theta(n) \ddot{\theta}(n) \\
& -m l \sin \theta(n) \dot{\theta}(n)^{2}=F(n)
\end{aligned}
$$

$m l^{2} \ddot{\theta}(n)+m l \cos \theta(n) \ddot{p}(n)+m g l \sin \theta(n)=0$,

where $n=1, \ldots, N ; \ddot{p}(n)$ and $F(n)$ represent acceleration and overall force at the $n$th sample respectively. $\theta(n), \dot{\theta}(n)$ and $\ddot{\theta}(n)$ are measured swing angle, swing velocity and swing acceleration at the $n$th sample respectively. At the period $[n-1, n)$, the overall force $F(n)$ is composed of the actuating force $F_{a}(n)$, the friction $F_{r}(n)$ and the disturbance $d(n)$ as

$F(n)=F_{a}(n)-F_{r}(n)+d(n)$.

In Eq. (12), $F_{r}(n)$ is the friction at the $n$th sample, which can be formulated similarly with Eq. (4) as

$F_{r}(n)=f_{r 0}(\tanh v(n) / \xi)+k_{p} v(n)+k_{r}|v(n)| v(n)$,

where $v(n)=\dot{p}(n)$ is the velocity of trolley at the $n$th sample.

Note that the discrete-time model is nonlinear. For applying our proposed linear MPC approach, a linear discrete steady-space model is required. Based on continuous steady-space model Eq. (9), the discrete statespace equation can be deduced using basic control theory as

$\left\{\begin{array}{l}x(n+1)=G x(n)+H u(n) \\ y(n)=x(n)\end{array}\right.$,

where $x(n) \triangleq\left[p(n)-p_{d}, \dot{p}(n), \theta(n)\right.$ and $u(n) \triangleq \ddot{p}(n) . G$ and $H$ are the system matrix and input matrix in the state-space as

$G=\left[\begin{array}{cccc}1 & t_{0} & 0 & 0 \\ 0 & 1 & 0 & 0 \\ 0 & 0 & \cos w_{n} t_{0} & \frac{\sin w_{n} t_{0}}{w_{n}} \\ 0 & 0 & -w_{n} \sin w_{n} t_{0} & \cos w_{n} t_{0}\end{array}\right]$

$H=\left[0.5 t_{0}^{2}, t_{0}, \frac{\cos w_{n} t_{0}-1}{l w_{n}^{2}},-\frac{\sin w_{n} t_{0}}{l w_{n}}\right]^{T}$
Note that the countability matrix $\left[H G H G^{2} H G^{3} H\right]$ has full rank. There exists optimal design of controller that ensures system stable at $x=0$.

In the discrete model, we denote the vector of acceleration as $\boldsymbol{a}(a(n)=\ddot{p}(n))$, and denote the state vector of velocity as $\boldsymbol{v}(v(n)=\dot{p}(n))$. Suppose that the initial position is $p(0)$, the initial velocity is $v(0)$, the initial acceleration is $a(0)$, the initial swing angle is $\theta(0)$, and the initial swing velocity is $\dot{\theta}(0)$. Given an acceleration vector $\boldsymbol{a}$, from Eq. (14) the velocity $\boldsymbol{v}$ and the displacement $\boldsymbol{p}$ can be expressed as

$\left\{\begin{array}{l}\boldsymbol{p}=\boldsymbol{p}_{0}+B v(0) t_{0}+A_{p} \boldsymbol{a} t_{0}^{2} \\ \boldsymbol{v}=\boldsymbol{v}_{0}+A_{v} \boldsymbol{a} t_{0}\end{array}\right.$,

where

$\left\{\begin{array}{l}\boldsymbol{p}=[p(1), \ldots, p(N)]^{T} \\ \boldsymbol{v}=[v(1), \ldots, v(N)]^{T} \\ \boldsymbol{a}=[a(1), \ldots, a(N)]^{T}\end{array}\right.$

$\boldsymbol{p}_{0}=\overbrace{[p(0), \ldots, p(0)}^{N}]^{T}, \boldsymbol{v}_{0}=\overbrace{[v(0), \ldots, v(0)}^{N}]^{T}$,

$B=[1,2, \ldots, N]^{T}$,

$A_{p}=\left[\begin{array}{ccccc}0.5 & 0 & 0 & \ldots & 0 \\ 1.5 & 0.5 & 0 & \ldots & 0 \\ 2.5 & 1.5 & 0.5 & \ldots & 0 \\ \vdots & \vdots & \vdots & \vdots & \vdots \\ N-0.5 & N-1.5 & N-2.5 & \ldots & 0.5\end{array}\right]_{N \times N}$

$A_{v}=\left[\begin{array}{ccccc}1 & 0 & 0 & \ldots & 0 \\ 1 & 1 & 0 & \ldots & 0 \\ 1 & 1 & 1 & \ldots & 0 \\ \vdots & \vdots & \vdots & \vdots & \vdots \\ 1 & 1 & 1 & \ldots & 1\end{array}\right]_{N \times N}$

According to Eq. (14), the swing angle $\theta(n)$ can be formulated as

$\theta(n)=\theta(0) \cos \left(n w_{n} t_{0}\right)+\frac{\dot{\theta}(0)}{w_{n}} \sin \left(n w_{n} t_{0}\right)+A_{\theta} \boldsymbol{a}$,

where

$A_{\theta}=\frac{1}{l w_{n}^{2}}\left[\begin{array}{c}\cos N w_{n} t_{0}-\cos (N-1) w_{n} t_{0} \\ \cos (N-1) w_{n} t_{0}-\cos (N-2) w_{n} t_{0} \\ \cos (N-2) w_{n} t_{0}-\cos (N-3) w_{n} t_{0} \\ \vdots \\ \cos 2 w_{n} t_{0}-\cos 3 w_{n} t_{0} \\ \cos w_{n} t_{0}-1\end{array}\right]$. 
In Eq. (23), the first two components at the right hand side is the initial-condition response and the third component is the forced response. It can be noticed that Eq. (17) and Eq. (23) show calculation to predict state variables from the initial time. From the $k$ th sample, $x(k+i), 1 \leq i \leq N-k$ has similar expression with Eq. (17) and Eq. (23) according to the discrete statespace equation.

In our proposed MPC, the input sequence is the actuating force over the transportation period. In the above discrete model, when acceleration is determined, force and velocity can be determined at the same time. Therefore, for the cranes with actuating motors working in the force or velocity control mode, the discrete model can be used. In other words, acceleration is equivalent with force and velocity using simple transformations according to equations (10) and (17). Based on this discrete model, the procedure of the MPC approach is given at the following section. Note that the discrete model is still useful in other control strategies, such as optimal control, proportional integral derivative (PID) control, and some closed-loop control methods.

\section{Model Predictive Control Approach}

Before introducing the MPC approach, the new objective function in terms of energy efficiency and safety must be formulated. Note that the control period $t_{c}$ is usually same or larger than the sampling period $t_{0}$ in the MPC. For the interval $\left[k, K\right.$ ) (where $K=T / t_{c}$ and $0 \leq k<K$ is the current instant), energy consumption can be expressed as

$E=\int_{k t_{c}}^{T} F_{a} \dot{x} d t=\sum_{i=k+1}^{K} F_{a}(i) v(i) t_{c}$

where $E$ is energy consumption of the motor; $F_{a}(i)$ and $v(i)$ are the actuating force and the velocity at the control period $[i-1, i)$. Substituting Eq. (10) into Eq. (12), $F_{a}(n)$ can be expressed by $a(n)$.

For the interval $[k, N)$, safety can be evaluated by the maximal swing angle as

$\theta_{s}=\max _{i \in\{k+1, \ldots, K\}} \theta(i)$,

where $S$ is the maximal swing angle in the future interval. According to Eq. (23), the swing angle can also be expressed by $a(i)$.

The proposed objective function has integrated energy consumption and safety as
$J=\alpha \sum_{i=k+1}^{K} F_{a}(i) v(i) t_{c}+(1-\alpha) \max _{i \in\{k+1, \ldots, K\}} \theta(i)$,

where $\alpha$ is the weighting parameter for integration. As energy efficiency and safety can be expressed by acceleration, the objective function value is only determined by $a(i)$.

The control input $a(i)$ must be bounded by the maximal acceleration $a_{m}$ as

$a(i) \in\left[-a_{m}, a_{m}\right], i=1, \ldots, K$.

There are many equality and inequality constraints for the objective function. Equality constraints include final states of displacement, velocity, and swing angle. Inequality constraints include limitation of the maximal velocity, the maximal acceleration, and the maximal swing. Therefore, the objective function (27) must be subject to a set of constraints as

$\left\{\begin{array}{l}p(K)=p_{d} \\ v(K)=0 \\ \theta(K)=0 \\ \theta(K-1)=0 \\ |a(i)| \leq a_{m}, \quad i=k+1, \ldots, K \\ 0 \leq v(i) \leq v_{m}, i=k+1, \ldots, K \\ |\theta(i)| \leq \theta_{m}, \quad i=k+1, \ldots, K\end{array}\right.$

MPC is employed to solve this optimal control problem at each control period $t_{c}$, but not in each sampling period $t_{0}\left(t_{0}<<t_{c}\right)$ for saving computational cost. In the proposed MPC approach, the optimal control problem in the prediction horizon $[k, K)$ is repeatedly solved $(k=0,1, \ldots, K)$. The input is applied to the system based on the obtained optimal solution. The optimal control problem, including the objective function and the set of constraints, has been defined in Eq. (27) and (29). The optimization variable is the sequence of the acceleration for each control period. At the $k$ th sample, an optimal solution of acceleration $[a(k+1), a(k+2), \ldots, a(K)]^{T}$ can be obtained after solving the optimal problem. According to the first part of solution, the actuating force $F(k+1)$ is computed and applied to the system in the next control period $t_{c}$, i.e., $\left[k t_{c}, k t_{c}+t_{c}\right)$. The procedure of the MPC approach can be illustrated as Algorithm 1.

It can be noticed that in the MPC approach reference trajectories are not required, and extra computation of planning trajectory is skipped. At each sampling instant, the horizon of the optimal control problem will be decreased by one when approaching the end. 


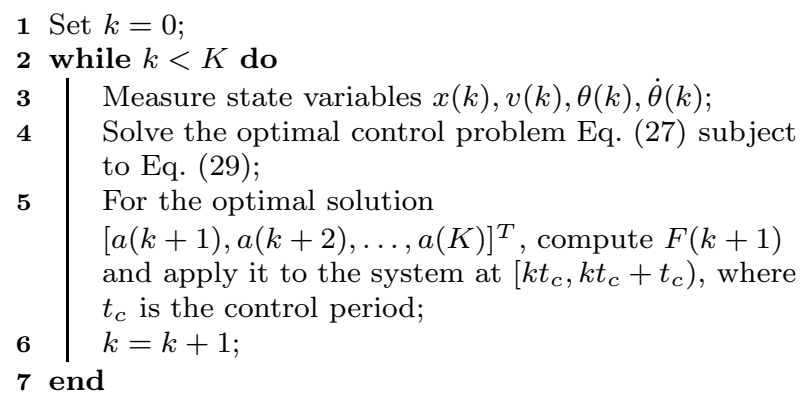

Algorithm 1: The proposed MPC approach for crane control

At the beginning of each control period, displacement, velocity, swing angle and swing velocity are measured. If there are disturbances in the previous periods, they can be detected. The optimal MPC controller will make the compensation and correction automatically. For this reason, the closed-loop nature of the MPC controller comes with an inherent property of robustness.

\section{Numerical Simulation}

The overhead crane system described in [28] is used to test our proposed MPC approach. The physical parameters of the system are listed as follows

$m=1.025 \mathrm{~kg}, M=7 \mathrm{~kg}, l=0.75 \mathrm{~m}, g=9.8 \mathrm{~m} / \mathrm{s}^{2}$.

The desired location of trolley in simulation is set as $p_{d}=0.6 \mathrm{~m}$, and the practical constraints are given as

$v_{m}=0.4 \mathrm{~m} / \mathrm{s}, a_{m}=0.2 \mathrm{~m} / \mathrm{s}^{2}, \theta_{m}=5^{\circ}$.

The parameters for the friction model Eq. (4) are referred from the offline regression results in [29] as

$f_{r 0}=4.4, k_{p}=0.05 ; k_{r}=0.45, \xi=0.01$.

For comparisons, we also evaluate the open-loop optimal control method on the tested crane. The openloop optimal controller utilizes the optimal trajectory in terms of energy consumption and safety, which is planned before start.

In this simulation section, zeros initial conditions are assumed as $p(0)=0, \dot{p}(0)=0, \ddot{p}(0)=0, \dot{\theta}(0)=$ $0, \ddot{\theta}(0)=0$. The MPC approach is implemented as the block diagram shown in Fig. 2. At each sampling period, the optimal acceleration $a(k)$ is obtained by minimizing the objective (27) based on states $x(k), v(k)$, $\theta(k)$, and $\dot{\theta}(k)$. Note that these states variables can be measured by sensors in practical applications. Here the state variables are calculated based on the system simulation when the disturbance $d(k)$ is assumed known. In the following simulation, the evaluated control period is $7 \mathrm{~s}$; the sampling period $t$ is $0.0005 \mathrm{~s}$; and the control period is 0.1s. In the MPC and the open-loop control, the optimization algorithm for minimizing the objective function is chosen as fmincon function in the Matlab software. In the fmincon function, the algorithm type is set as "active-set" and the maximum function evaluation times are 7000. In this simulation, we will validate stability and robustness of the MPC approach in the presence of different types of disturbances, including the random disturbances, the impulse disturbances, the periodical sine-wave disturbances. The disturbances are added to the actuating force between $1 \mathrm{~s}$ and $3 \mathrm{~s}$ as shown in Fig. 3.
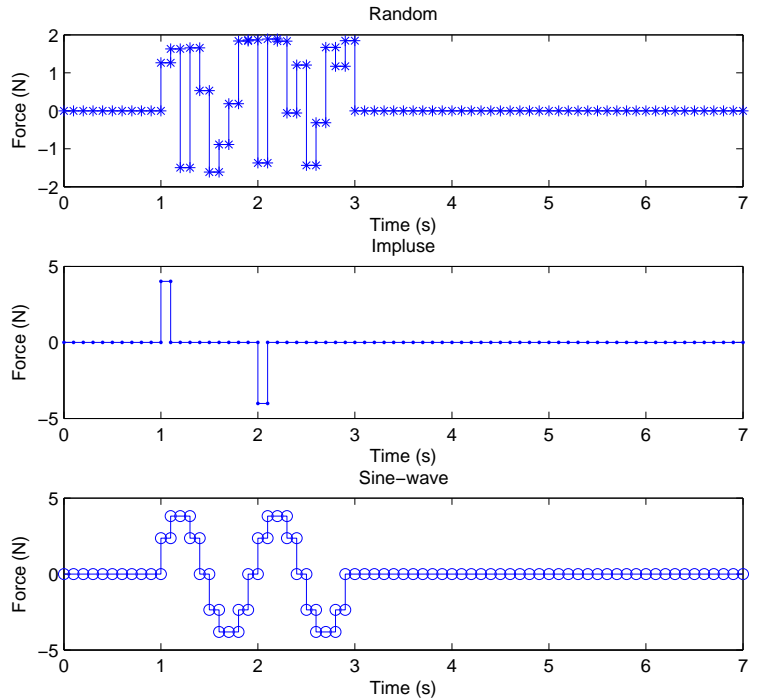

Fig. 3 Three types of disturbances

\subsection{Tests on energy efficiency $(\alpha=1)$}

When $\alpha=1$ is used in Eq. (27), energy consumption will only be considered over the evaluation time. When there exists no disturbance, the results of openloop control and MPC have been shown in Fig. 4. For the open-loop control, energy consumption is $2.6381 \mathrm{~J}$. For the MPC, energy consumption is $2.6378 \mathrm{~J}$. Fig. 4 shows profiles of acceleration and state variables (displacement, velocity and swing). It can be noticed that these profiles obtained by the MPC and the open-loop control are close to each other when no disturbance exists.

For the random disturbances, energy consumption obtained in the MPC is $2.6593 \mathrm{~J}$; energy consumption 


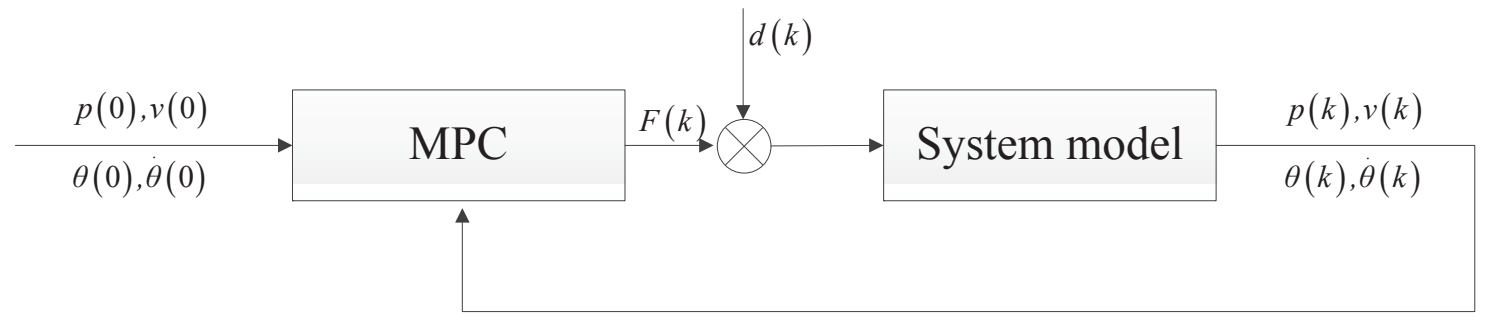

Fig. 2 Block diagram of the MPC approach
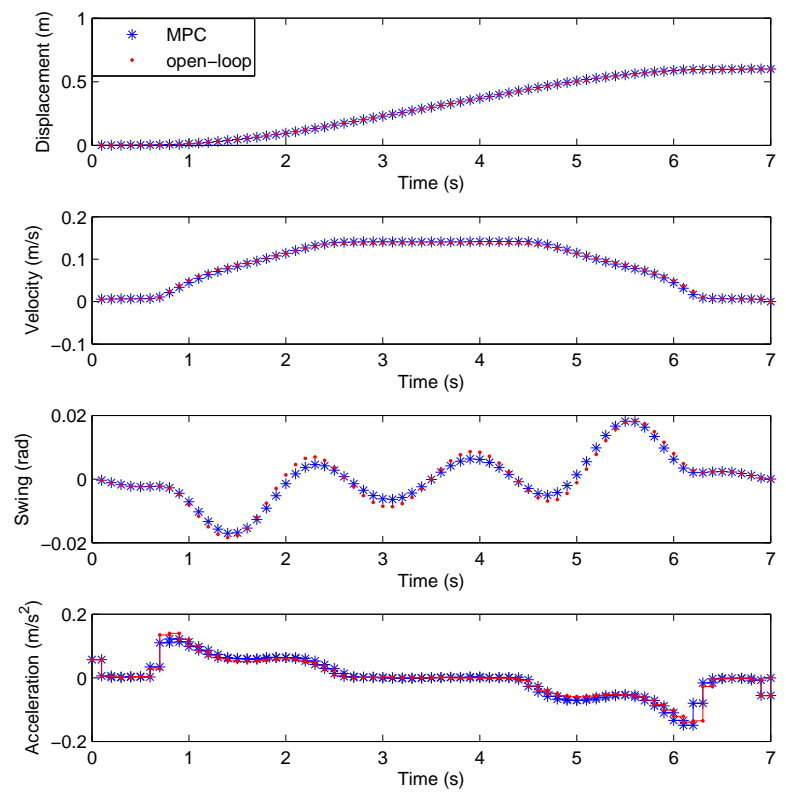

Fig. 4 Comparison between the MPC and the open-loop control with no disturbance $(\alpha=1)$

obtained in the open-loop control is $5.8463 \mathrm{~J}$. The MPC is more energy efficient than the open-loop control. Fig. 5 gives profiles of state variables of these two approaches between $5 \mathrm{~s}$ and $7 \mathrm{~s}$ with the random disturbances. Nominal profiles which represent optimal profiles under the assumption of no disturbance are also given in the figure. It is worth noting that the final displacement, velocity and swing in the open-loop control violate the practical constraints. The results of the MPC approach can satisfy the constraints, such as zero velocity and zero swing. The MPC profiles can converge to the nominal profiles at the end of transportation period.

For the impulse disturbances, energy consumption obtained in the MPC is $2.6584 \mathrm{~J}$; energy consumption obtained in the open-loop control is $2.8834 \mathrm{~J}$. Energy consumed in the open-loop approach is more than energy consumption in the MPC due to the impulse disturbances. Fig. 6 gives profiles of state variables of the
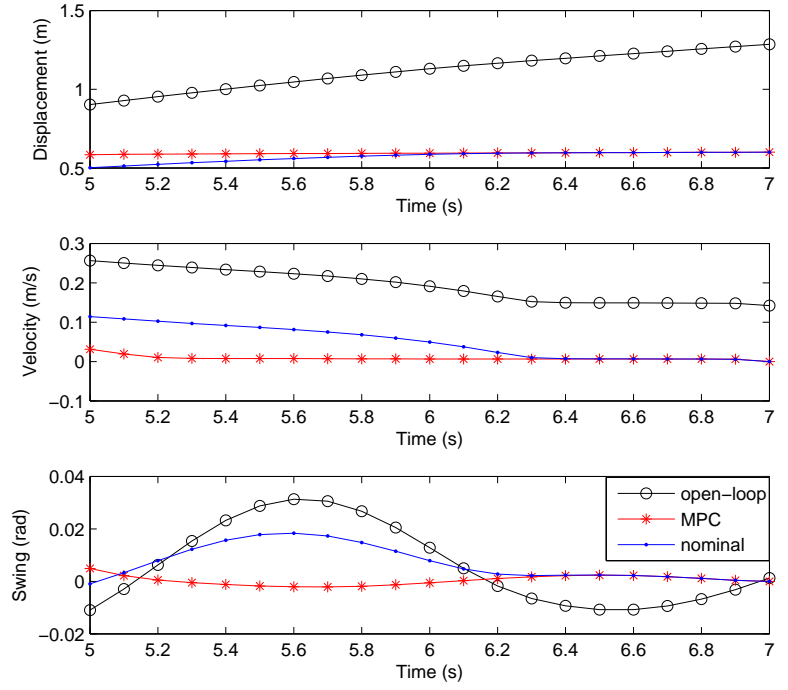

Fig. 5 State variables of the MPC and the open-loop control with the random disturbance $(\alpha=1)$

MPC and open-loop approaches between $5 \mathrm{~s}$ and $7 \mathrm{~s}$. In the open-loop approach, the final displacement, velocity and swing violate the practical constraints. In the MPC, the results obtained can satisfy the constraints, such as zero velocity and zero swing. The MPC profiles are more closer to the nominal profiles and converge at the end.

For the sine-wave disturbances, energy consumption obtained in the MPC is also $2.7394 \mathrm{~J}$; energy consumption obtained in the open-loop control is $3.4252 \mathrm{~J}$. The open-loop approach has less energy consumption than the MPC due to the periodic disturbances. Fig. 7 shows their profiles of state variables between $5 \mathrm{~s}$ and $7 \mathrm{~s}$ with the sine-wave disturbance. The same conclusion can be drawn in the open-loop control that final displacement, velocity and swing violate the practical constraints. The results obtained by the MPC can satisfy the constraints, such as zero final velocity and zero final swing.

It can be concluded that for different kinds of disturbances the MPC can reduce energy consumption of crane to an acceptable extent while the practical con- 

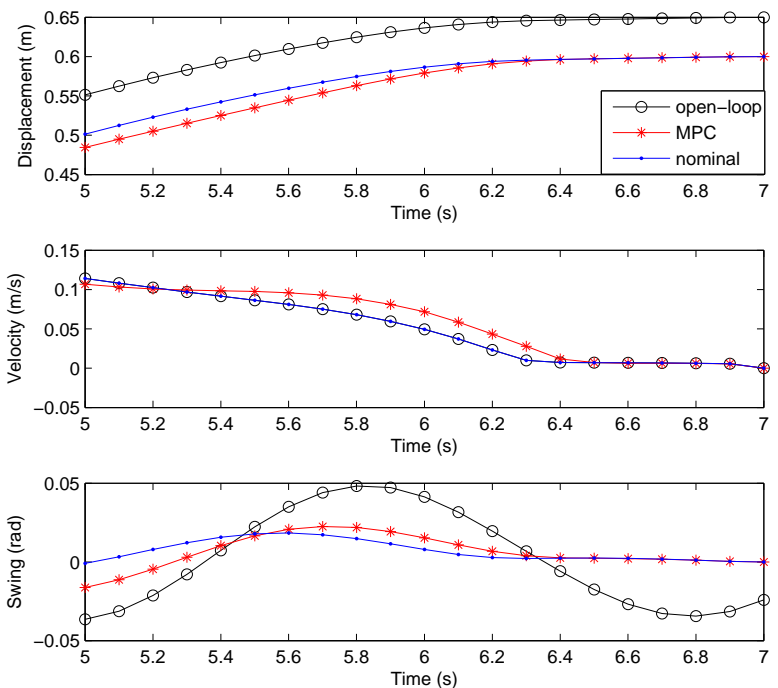

Fig. 6 State variables of the MPC and the open-loop control with the impulse disturbance $(\alpha=1)$
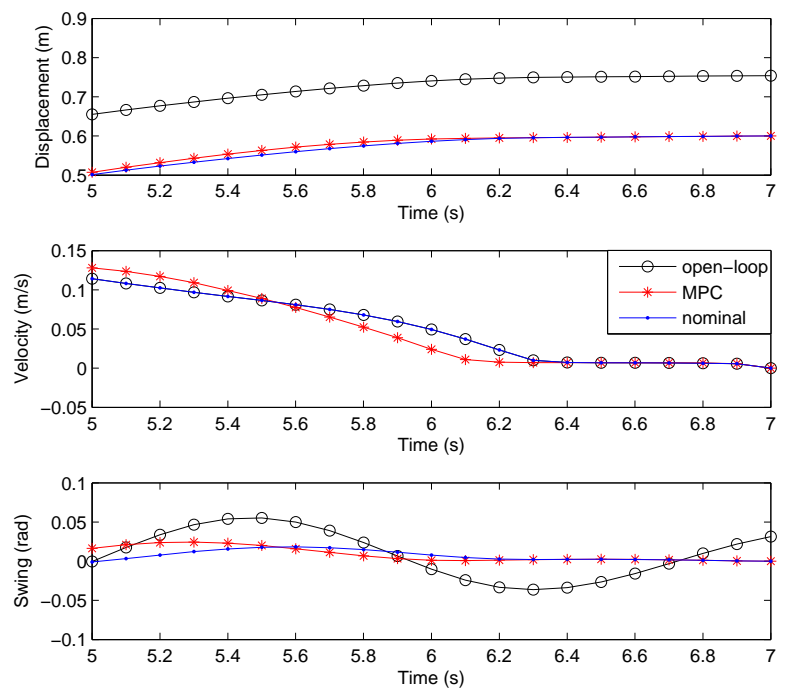

Fig. 7 State variables of the MPC and the open-loop control with the sine-wave disturbance $(\alpha=1)$

straints can all be satisfied. As mentioned before, in the MPC as the constraints are satisfied, the payload can be lowered immediately without any adjustment. For the open-loop control, the required adjustment asks for extra energy and time, which can be saved in our proposed MPC approach.
5.2 Tests on safety $(\alpha=0)$

When $\alpha=0$, safety is considered in the objective function for minimizing the maximal swing angle. When there exists no disturbance, the solutions obtained in the open loop control and the MPC have the same maximal swing angles 0.0062 rad. Besides the maximal swing angle, the residual swing angle is another important metric to evaluate the oscillation of the payload. In this paper, the residual swing is defined as the average swing angle in the last $R$ seconds $(R=2$ is used here). The residual swing in the open-loop control is $0.0062 \mathrm{rad}$; the residual swing in the MPC is $0.0057 \mathrm{rad}$. The MPC approach can obtain less residual swing angle than the optimal solution. The reason is that when the crane is approaching the destination, each optimal control problem in the MPC is to minimize the residual oscillation at each control period. Fig. 8 gives profiles obtained by the MPC and the open-loop control in this case. It can be noticed that the maximal swing angles in the MPC and the open-loop control are the same. For reducing the residual swing, accelerations in the last $2 \mathrm{~s}$ have large differences between the MPC and the open-loop control.

For the three kinds of disturbances, the maximal and residual swing angles obtained in the MPC and open-loop approaches are listed in Table 1. The Results of the MPC are better than those of the open-loop control in terms of small maximal and residual swing. For the random disturbances, the average of maximal and residual swing is $0.0077 \mathrm{rad}$ for the MPC and 0.0131 rad for the open-loop control. For the impulse disturbances, the maximal swing $0.0141 \mathrm{rad}$ and the residual swing $0.0068 \mathrm{rad}$ are obtained in the MPC. The average of them is smaller than the average obtained by the open-loop control. The same conclusion can be found in the case of the sine-wave disturbances. Fig. 9, 10 and 11 give the profiles of displacement, velocity and swing between $5 \mathrm{~s}$ and $7 \mathrm{~s}$ for the three kinds of disturbances respectively. Figures indicate the practical constraints can be satisfied in the MPC approach and the MPC profiles can converge to the nominal profiles.

It can be concluded that for different kinds of disturbances the safety of operation is enhanced in the MPC by minimizing the maximal and residual swing while all the practical constraints are satisfied. Compared with the open-loop control, the MPC does not need extra energy and time to adjust the violated constraints. Furthermore, the MPC approach aims to minimize the maximal swing, but due to its specific structure it reduces the residual swing as well. 
Table 1 Swing comparisons of different disturbances $(\alpha=0)$

\begin{tabular}{|c|c|c|c|c|c|c|}
\hline & \multicolumn{2}{|c|}{ Random } & \multicolumn{2}{c|}{ Impulse } & \multicolumn{2}{c|}{ Sine-wave } \\
\cline { 2 - 7 } & MPC & open-loop & MPC & open-loop & MPC & open-loop \\
\hline Maximal swing (rad) & 0.0097 & 0.0203 & 0.0141 & 0.0413 & 0.0513 & 0.0610 \\
\hline Residual swing (rad) & 0.0056 & 0.0084 & 0.0068 & 0.0238 & 0.0148 & 0.0245 \\
\hline Average (rad) & $\mathbf{0 . 0 0 7 7}$ & 0.0131 & $\mathbf{0 . 0 1 0 4}$ & 0.0326 & $\mathbf{0 . 0 3 3 0}$ & 0.0427 \\
\hline
\end{tabular}

Table 2 General comparisons of different disturbance $(\alpha=0.01)$

\begin{tabular}{|c|c|c|c|c|c|c|}
\hline & \multicolumn{2}{|c|}{ Random } & \multicolumn{2}{c|}{ Impulse } & \multicolumn{2}{c|}{ Sine-wave } \\
\cline { 2 - 7 } & MPC & open-loop & MPC & open-loop & MPC & open-loop \\
\hline Objective value & $\mathbf{0 . 0 3 6 4}$ & 0.0787 & $\mathbf{0 . 0 4 0 0}$ & 0.0698 & $\mathbf{0 . 0 7 9 3}$ & 0.0946 \\
\hline Energy consumption (J) & 2.6839 & 5.8530 & 2.6818 & 2.8920 & 2.8483 & 3.4267 \\
\hline Maximal swing (rad) & 0.0096 & 0.0204 & 0.0133 & 0.0413 & 0.0513 & 0.0610 \\
\hline Residual swing (rad) & 0.0057 & 0.0085 & 0.0068 & 0.0238 & 0.0148 & 0.0245 \\
\hline
\end{tabular}
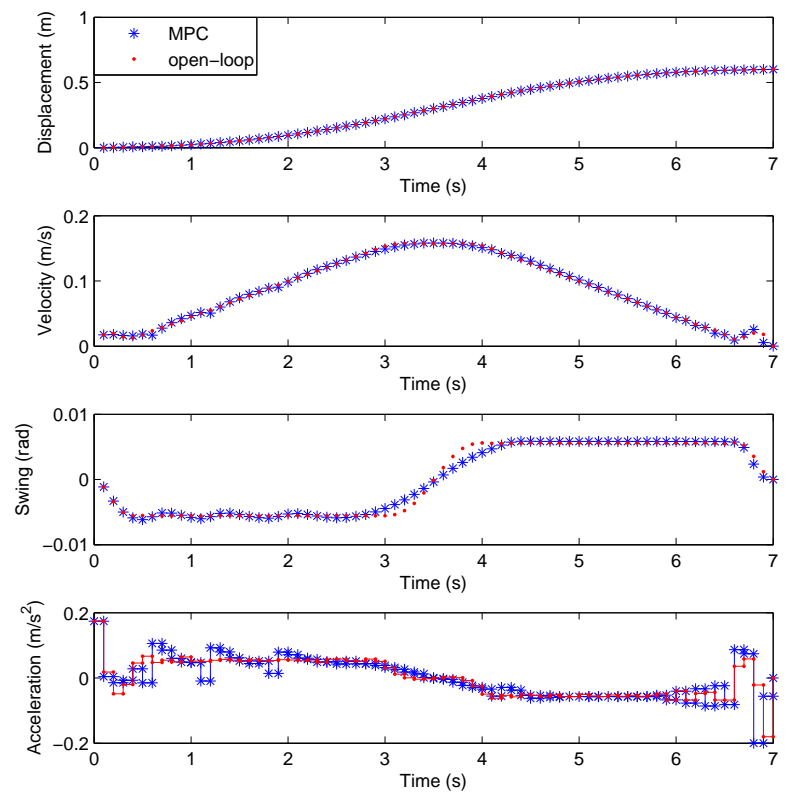

Fig. 8 Comparison between the MPC and the open-loop control with no disturbance $(\alpha=0)$

\subsection{Tests on energy efficiency and safety $(\alpha=0.01)$}

When $\alpha=0.01$, both energy efficiency and safety have been considered in the control objective. If no disturbance exists, the optimal solution obtained in the MPC has $J=0.0324(E=2.6412, S=0.0060)$, while the solution obtained in the open-loop control has $J=$ 0.0326 ( $E=2.6437, S=0.0063)$. It can be noticed that the MPC is slightly better than the open-loop control in terms of energy consumption and safety.

For the three kinds of disturbances, we have compared the MPC with the open-loop control on the same crane system. Table 2 gives their results in terms of objective value, energy consumption, maximal swing, and
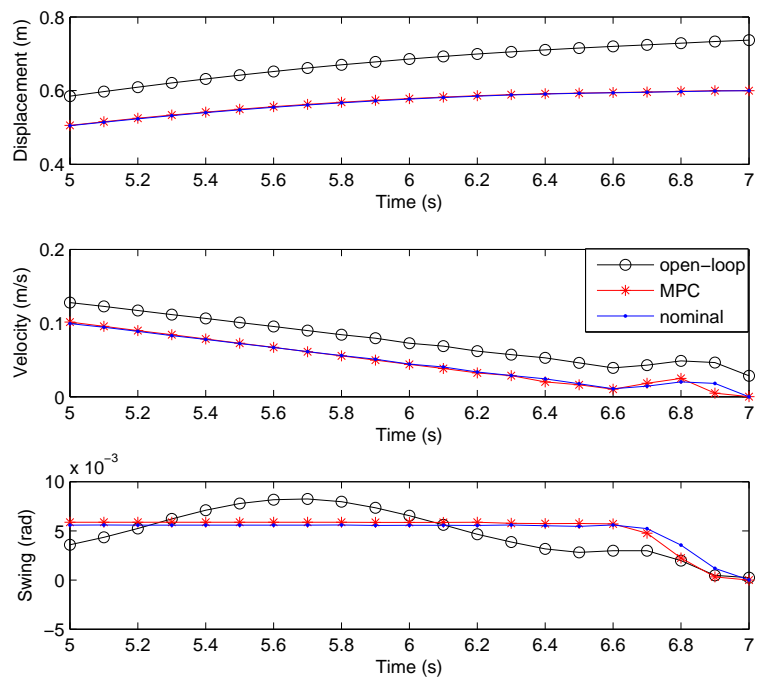

Fig. 9 State variables of the MPC and the open-loop with the random disturbance $(\alpha=0)$

residual swing. For the random, impulse and sine-wave disturbances, it can be noticed that the MPC performs much better than the open-loop control in terms of energy consumption and swing. Especially, the MPC is suitable to reduce the residual swing. Fig. 12, 13 and 14 are the profiles of displacement, velocity and swing between $5 \mathrm{~s}$ and $7 \mathrm{~s}$ when each kind of disturbances exist. As shown in figures, the open-loop control cannot ensure all practical constraints satisfied, but results obtained in the MPC can satisfy all constraints.

In addition, to validate the robustness of the proposed MPC approach against parameter variations, we examine the following tow extreme cases during the transportation process.

(1) The rope length is changed from $0.75 \mathrm{~m}$ to $1 \mathrm{~m}$ abruptly at $t=1 \mathrm{~s}$. 

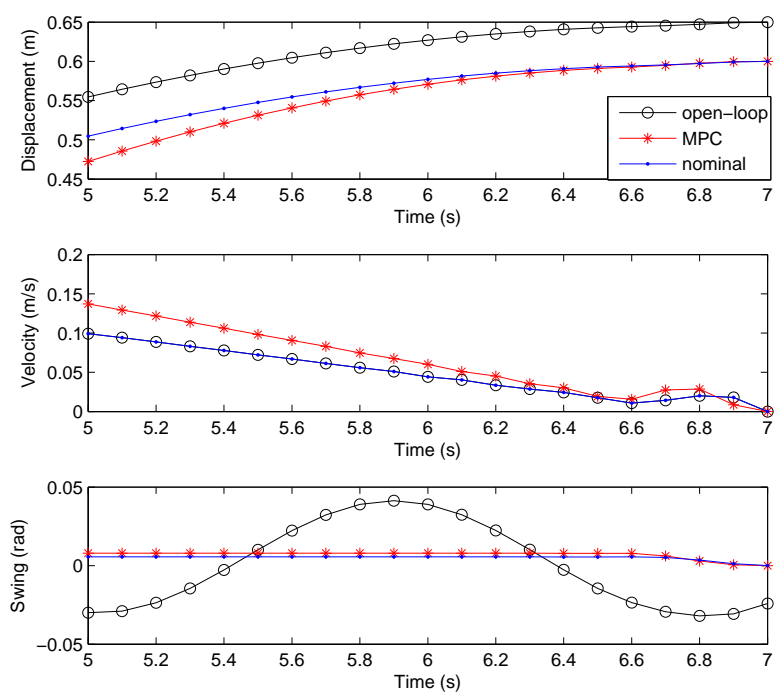

Fig. 10 State variables of the MPC and the open-loop control with the implulse disturbance $(\alpha=0)$
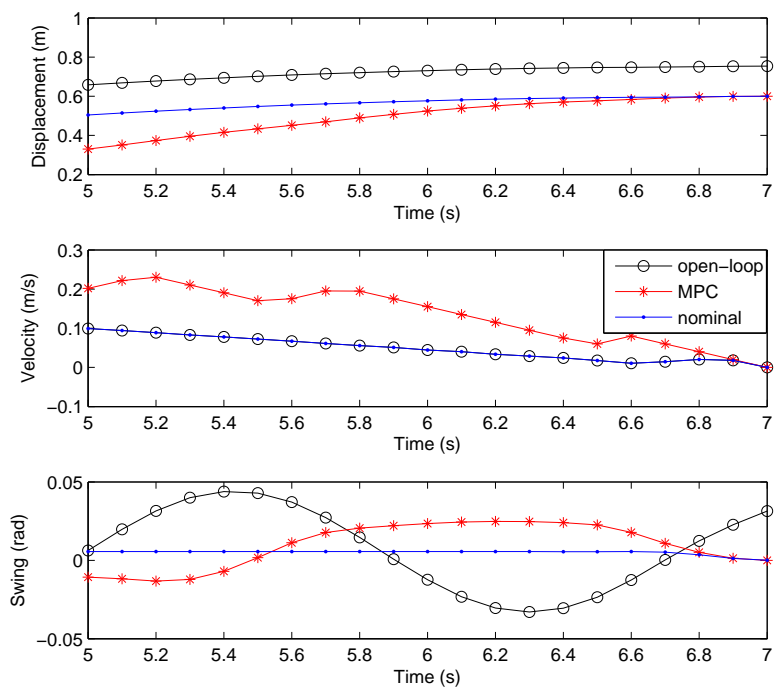

Fig. 11 State variables of the MPC and the open-loop control with the sine-wave disturbance $(\alpha=0)$

(2) The payload mass is increased from $1.025 \mathrm{~kg}$ to $1.2 \mathrm{~kg}$ abruptly at $t=4 \mathrm{~s}$.

Note that abrupt changes of parameter are much tougher situations than time variation of parameter. In these two cases, the crane can be controlled to arrive the end without violation of terminate constraints. In Case 1 , the energy consumption is $2.6488 \mathrm{~J}$, and the maximal swing is $0.0060 \mathrm{rad}$. In Case 2, the energy consumption will be increased due to the payload mass change. The energy consumption is $2.6494 \mathrm{~J}$, and the maximal swing is $0.0060 \mathrm{rad}$. The profiles resulted from constant pa-
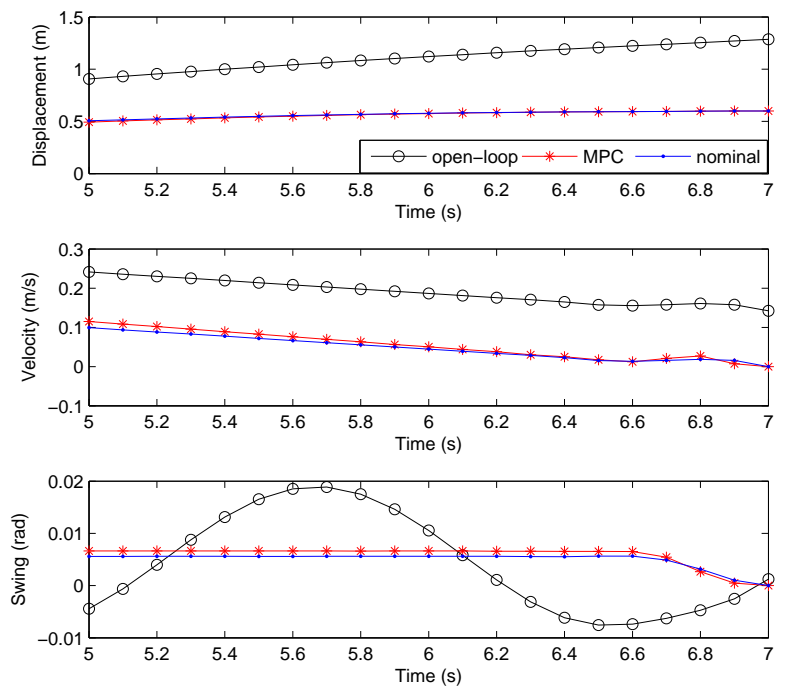

Fig. 12 State variables of the MPC and open-loop approaches with the random disturbance $(\alpha=0.01)$
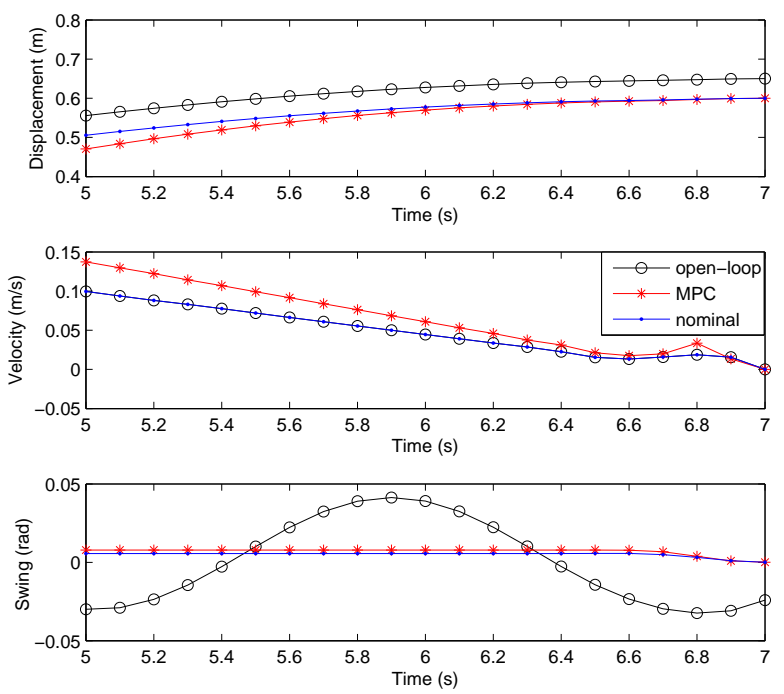

Fig. 13 State variables of the MPC and open-loop approaches with the impluse disturbance $(\alpha=0.01)$

rameters and changed parameters have been given in Fig. 15 and 16. In Case 1, the acceleration profile has been adapted according to the change of rope length after $1 \mathrm{~s}$. In Case 2, the acceleration profile has been adapted according to the change of mass after $4 \mathrm{~s}$. It can be concluded that under different situations of varied parameters the MPC approach is useful to achieve the transportation task with good operational performance. The trolley accurately reaches the destination with zero final swing. During the transportation, the energy consumption is optimized, and the maximal swing 

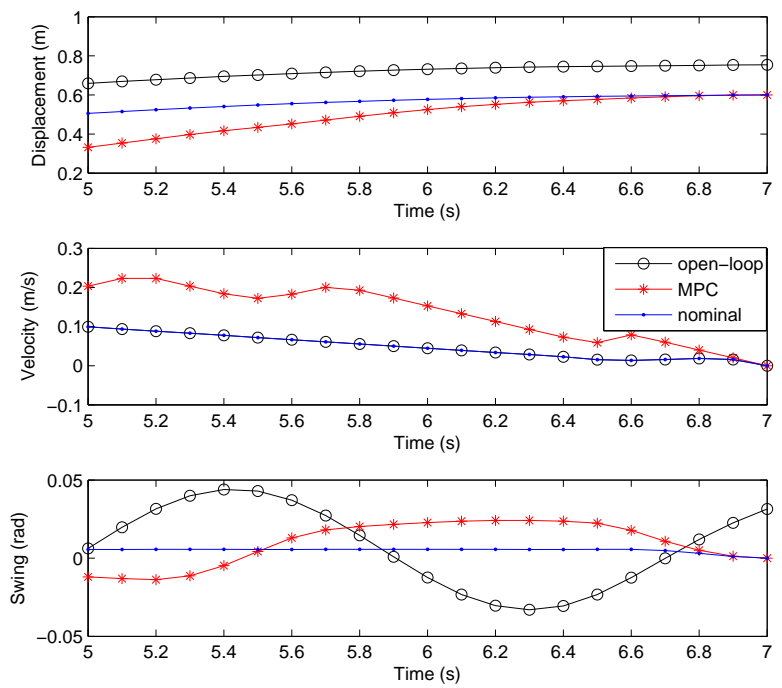

Fig. 14 State variables of the MPC and open-loop approaches with the sine-wave disturbance $(\alpha=0.01)$

as well as the residual swing has been suppressed within an acceptable range.
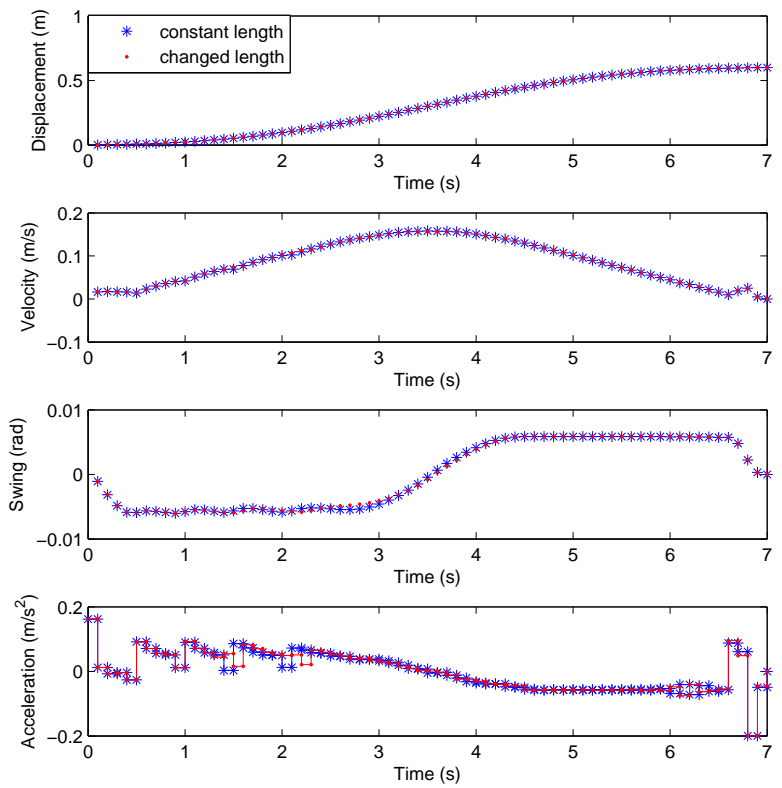

Fig. 15 Case 1: profiles obtained in the MPC when the rope length is changed in comparison with constant length

It can be concluded that the MPC can minimize the energy consumption and maximize the operation safety at the same time in our proposed approach. Whether disturbances exist or not, the MPC can be used to find
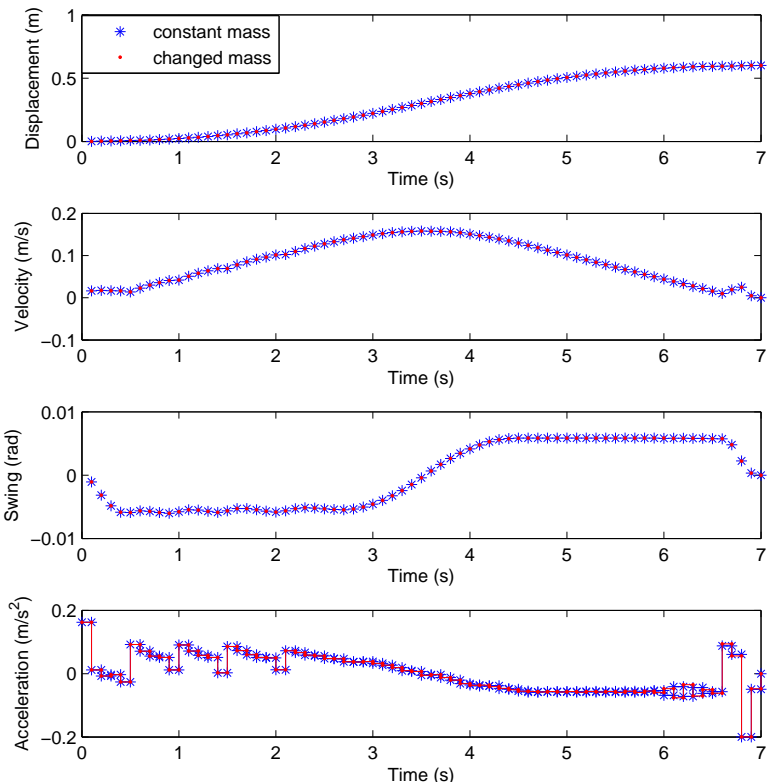

Fig. 16 Case 2: profiles obtained in the MPC when the payload mass is changed in comparison with the constant mass

the close-optimal results. The practical constraints are reliably satisfied over the control horizon.

\subsection{Comparison with other control methods}

To validate whether the performance improvement of the proposed approach is significant, some commonly used crane control methods have been selected in the comparison study. They all belong to energy-based feedback control, in which the highly coupled and complicated underactuated system dynamics can be conventional analyzed via the system energy to achieve satisfactory control performances. A series of energy-based controller, including $E^{2}$ controller [6], trolley/gantry kinetic energy (TKE) controller [4] and End-Effect Motion (EEM) based controller [27], have been proposed with similar forms of proportional-derivative (PD) control. The expressions of these controllers are simply illustrated as follows. More details can be referred from their original publications.

(1) $E^{2}$ control law:

$F_{E^{2}}=\frac{-\left(k_{p} e+k_{d} \dot{x}\right) m(\theta)-k v m \sin \theta\left(l \dot{\theta}^{2}+g \cos \theta\right)}{k_{E} m(\theta) E(t)+k_{v}}$, 
Table 3 Control performance comparison

\begin{tabular}{|c|c|c|c|c|c|}
\hline Controllers & $p_{f}(\mathrm{~m})$ & $\theta_{s}(\circ)$ & $\theta_{\text {res }}(\circ)$ & $t_{s}(\mathrm{~s})$ & $F_{a}^{\max }(\mathrm{N})$ \\
\hline$E^{2}$ controller & 0.601 & 4.86 & 1.59 & 14.10 & 23.10 \\
\hline TKE controller & 0.597 & 3.70 & 1.40 & 11.68 & 14.67 \\
\hline EEM controller & 0.599 & 3.19 & 0.25 & 4.18 & 11.32 \\
\hline MPC controller & 0.6 & 0.344 & 0 & 7 & 1.363 \\
\hline
\end{tabular}

where $e=p-p_{d}$ is the displacement error, $E(t)$ is the system mechanical energy and $m(\theta)=M+m \sin ^{2} \theta$. $k_{p}, k_{d}, k_{E}, k_{v}$ are control gains of the $E^{2}$ controller.

(2) TKE control law:

$F_{T K E}=\frac{-k_{p} e-k_{d} \dot{x}+k_{v}[\zeta(\theta, \dot{\theta})-m \sin \theta \cos \theta \dot{\theta} \dot{x}]}{k_{E}+k_{v}}$,

where $\zeta(\theta, \dot{\theta})=-m \sin \theta\left(l \dot{\theta}^{2}+g \cos \theta\right)$, and $k_{p}, k_{d}, k_{E}, k_{v}$ are control gains of the TKE controller.

(3) EEM control law:

$F_{E E M}=-k_{p}\left(e-k_{a} \sin \theta\right)-k_{d}\left(\dot{e}-k_{a} \dot{\theta} \cos \theta\right)$,

where $k_{p}, k_{d}, k_{a}$ are control gains of the EEM controller.

These three controllers are implemented on the same crane system mentioned before. For these controllers, parameter settings are following the settings given in [27]. The proposed MPC controller has been compared with the three controllers. For each controller, the performance is evaluated in terms of the final displacement $p_{f}$, the maximal swing $\theta_{s}$, the residual swing $\theta_{\text {res }}$, the arriving time $t_{s}$ and the maximal actuating force $F_{a}^{\max }$. Table 3 lists experimental results of $E^{2}$, TKE, EEM and the proposed MPC approaches for crane control. In the MPC, a constraint optimization problem is solved in each receding horizon. Therefore, more terminal constraints can be satisfied in the MPC approach than in the compared control methods. For example, the final displacement obtained in the MPC is $p_{d}=0.6$ and the residential swing in the $\mathrm{MPC}$ is zero. As shown in the table, the MPC performs the best in terms of zero residual swing, the smallest maximum swing and the smallest actuating force. Although the EEM controller can achieve the shortest arriving time with great time efficiency, it cannot strictly follow constants considered in this paper, such as constraints of maximal swing, maximal acceleration and velocity. When the maximal actuating force is small, the actuating motor works with smoothly accelerating or decelerating, which means that motor oscillation as well as energy consumption can be reduced. The smallest actuating force obtained in the MPC indicates some reason of the resulted energy efficiency.

\section{Conclusion}

For overhead cranes, energy consumption and safety have been modeled to achieve the control performance improvement. Using the model predictive control (MPC) approach, the crane can be controlled to arrive the destination with good performance of energy efficiency and safety, because energy consumption and maximal swing angle have been minimized during the horizontal transportation. To simulate real world applications with disturbances, three kinds of disturbances (random, impulse and sine-wave) have been tested in the proposed MPC approach. From numerical results obtained, it has been shown that the MPC approach is stable and robust to find the close-optimal solutions when disturbances exist.

The MPC approach is suitable to solve the process control of transportation systems. For crane control, the MPC utilizes the information of current displacement, velocity, and swing angle for predicting the following control sequence (acceleration or force). As only the first sample of the sequence is applied, any disturbance that occurred in the system can be detected before the next control period. The MPC can correct the control variable accordingly for the next period. This is why the MPC can obtain accurate and robust results in crane control.

In this paper, the crane system is simplified as a deterministic model without consideration of system uncertainties, such as bridge deformation, time-varying rope length and payload weight. For complicated system with such uncertainties, the proposed approach still can be employed to pursuit minimal energy consumption and maximal safety if some stochastic MPC is chosen instead of the standard MPC and uncertainties can be detected or approximated. Due to the length limitation of this paper, the stochastic model has not been included in the proposed approach. Future work may evaluate the stochastic or nonlinear MPC on complicated crane systems with uncertainties.

\section{References}

1. Arnold, E., Sawodny, O., Neupert, J., Schneider, K.: Anti-sway system for boom cranes based on a model predictive control approach. In: 2005 IEEE International 
Conference Mechatronics and Automation, vol. 3, pp. 1533-1538 Vol. 3 (2005)

2. Benhidjeb, A., Gissinger, G.: Fuzzy control of an overhead crane performance comparison with classic control. Control Engineering Practice 3(12), 1687 - 1696 (1995)

3. Blajer, W., Dziewiecki, K., Kolodziejczyk, K., Mazur, Z.: Inverse dynamics of underactuated mechanical systems: A simple case study and experimental verification. Communications in Nonlinear Science and Numerical Simulation 16(5), 2265 - 2272 (2011)

4. Fang, Y., Dixon, W., Dawson, D., Zergeroglu, E.: Nonlinear coupling control laws for an underactuated overhead crane system. IEEE/ASME Transactions on Mechatronics 8(3), 418-423 (2003)

5. Fang, Y., Ma, B., Wang, P., Zhang, X.: A motion planning-based adaptive control method for an underactuated crane system. IEEE Transactions on Control Systems Technology 20(1), 241-248 (2012)

6. Fang, Y., Zergeroglu, E., Dixon, W., Dawson, D.: Nonlinear coupling control laws for an overhead crane system. In: Proceedings of the 2001 IEEE International Conference on Control Applications, 2001, pp. 639-644 (2001)

7. García, C.E., Prett, D.M., Morari, M.: Model predictive control: Theory and practice - survey. Automatica 25(3), $335-348$ (1989)

8. Garrido, S., Abderrahim, M., Gimenez, A., Diez, R., Balaguer, C.: Anti-swinging input shaping control of an automatic construction crane. IEEE Transactions on Automation Science and Engineering 5(3), 549-557 (2008)

9. Hoang, N.Q., Lee, S.G., Kim, J.J., Kim, B.S.: Simple energy-based controller for a class of underactuated mechanical systems. International Journal of Precision Engineering and Manufacturing 15(8), 1529 - 1536 (2014)

10. Hung, N., Robert, J., Jianguo, Z., David, L., Peter, M., Shoudong, H., Tuan, N., Russell, N., Kaili, W.: Model predictive control of gantry crane with input nonlinearity compensation (2009)

11. Kleinman, D.: An easy way to stabilize a linear constant system. IEEE Transactions on Automatic Control 15(6), 692-692 (1970)

12. Lee, H.H.: Motion planning for three-dimensional overhead cranes with high-speed load hoisting. International Journal of Control 78(15), 875-886 (2005)

13. Mahfouf, M., Kee, C.H., Abbod, M.F., Linkens, D.A.: Fuzzy logic-based anti-sway control design for overhead cranes. Neural Computing \& Applications 9(1) (2000)

14. Makkar, C., Hu, G., Sawyer, W.G., Dixon, W.: Lyapunov-based tracking control in the presence of uncertain nonlinear parameterizable friction. IEEE Transactions on Automatic Control 52(10), 1988-1994 (2007)

15. Masoud, Z.: Effect of hoisting cable elasticity on antisway controllers of quay-side container cranes. Nonlinear Dynamics 58(1-2), 129-140 (2009)

16. Masoud, Z., Nayfeh, A.: Sway reduction on container cranes using delayed feedback controller. Nonlinear Dynamics 34(3-4), 347-358 (2003)

17. Moon, M., Vanlandingham, H., Beliveau, Y.: Fuzzy time optimal control of crane load. In: Proceedings of the 35th IEEE Conference on Decision and Control, 1996, vol. 2, pp. 1127-1132 vol.2 (1996)

18. Nayfeh, N., Baumann, W.: Nonlinear analysis of timedelay position feedback control of container cranes. Nonlinear Dynamics 53(1-2), 75-88 (2008)

19. Ngo, Q.H., Hong, K.S.: Sliding-mode antisway control of an offshore container crane. IEEE/ASME Transactions on Mechatronics 17(2), 201-209 (2012)
20. Otomega, B., Marinakis, A., Glavic, M., Van Cutsem, T.: Model predictive control to alleviate thermal overloads. IEEE Transactions on Power Systems 22(3), 1384-1385 (2007)

21. Park, M., Chwa, D., Eom, M.: Adaptive slidingmode antisway control of uncertain overhead cranes with high-speed hoisting motion. Fuzzy Systems, IEEE Transactions on PP(99), 1-1 (2014). DOI 10.1109/TFUZZ.2013.2290139

22. Park, M.S., Chwa, D., Hong, S.K.: Antisway tracking control of overhead cranes with system uncertainty and actuator nonlinearity using an adaptive fuzzy slidingmode control. IEEE Transactions on Industrial Electronics 55(11), 3972-3984 (2008)

23. Piazzi, A., Visioli, A.: Optimal dynamic-inversion-based control of an overhead crane. IEE Proceedings of Control Theory and Applications 149(5), 405-411 (2002)

24. Qin, S., Badgwell, T.A.: A survey of industrial model predictive control technology. Control Engineering Practice 11(7), 733 - 764 (2003)

25. Raffo, G., Gomes, G., Normey-Rico, J., Kelber, C., Becker, L.: A predictive controller for autonomous vehicle path tracking. IEEE Transactions on Intelligent Transportation Systems 10(1), 92-102 (2009)

26. Singhose, W., Porter, L., Kenison, M., Kriikku, E.: Effects of hoisting on the input shaping control of gantry cranes. Control Engineering Practice 8(10), 1159 - 1165 (2000)

27. Sun, N., Fang, Y.: New energy analytical results for the regulation of underactuated overhead cranes: An endeffector motion-based approach. IEEE Transactions on Industrial Electronics 59(12), 4723-4734 (2012)

28. Sun, N., Fang, Y., Zhang, X., Yuan, Y.: Transportation task-oriented trajectory planning for underactuated overhead cranes using geometric analysis. IET Control Theory Applications 6(10), 1410-1423 (2012)

29. Sun, N., Fang, Y., Zhang, Y., Ma, B.: A novel kinematic coupling-based trajectory planning method for overhead cranes. IEEE/ASME Transactions on Mechatronics 17(1), 166-173 (2012)

30. Tuan, L., Lee, S.G., Dang, V.H., Moon, S., Kim, B.: Partial feedback linearization control of a three-dimensional overhead crane. International Journal of Control, Automation and Systems 11(4), 718-727 (2013)

31. Tuan, L., Moon, S.C., Lee, W., Lee, S.G.: Adaptive sliding mode control of overhead cranes with varying cable length. Journal of Mechanical Science and Technology 27(3), 885-893 (2013). DOI 10.1007/s12206-013-0204-x

32. Wu, X., He, X., Sun, N., Fang, Y.: A novel anti-swing control method for 3-d overhead cranes. In: Proceedings of the 2014 American Control Conference (ACC), 2014, pp. 2821-2826 (2014)

33. Xia, X., Zhang, J., Elaiw, A.: An application of model predictive control to the dynamic economic dispatch of power generation. Control Engineering Practice 19(6), $638-648(2011)$

34. Yang, J.H., Yang, K.S.: Adaptive coupling control for overhead crane systems. Mechatronics 17(2), 143 - 152 (2007)

35. Zhang, L., Zhuan, X.: Optimal operation of heavy-haul trains equipped with electronically controlled pneumatic brake systems using model predictive control methodology. IEEE Transactions on Control Systems Technology 22(1), 13-22 (2014) 